\title{
The interrelationship between ventilatory inefficiency and left ventricular ejection fraction in terms of cardiovascular outcomes in heart failure outpatients
}

Shyh-Ming Chen ( $\sim$ syming99@gmail.com )

Chang Gung Memorial Hospital Kaohsiung Branch https://orcid.org/0000-0001-5256-7429

Lin-Yi Wang

Chang Gung Memorial Hospital Kaohsiung Branch

Po-Jui Wu

Chang Gung Memorial Hospital Kaohsiung Branch

Mei-Yun Liaw

Chang Gung Memorial Hospital Kaohsiung Branch

Yung-Lung Chen

Chang Gung Memorial Hospital Kaohsiung Branch

An-Ni Chen

Chang Gung Memorial Hospital Kaohsiung Branch

Tzu-Hsien Tsai

Chang Gung Memorial Hospital Kaohsiung Branch

Chi-Ling Hang

Chang Gung Memorial Hospital Kaohsiung Branch

Meng-Chih Lin

Chang Gung Memorial Hospital Kaohsiung Branch

Research article

Keywords: Heart failure, mortality, ejection fraction, cardiopulmonary exercise test, ventilatory inefficiency

Posted Date: June 2nd, 2020

DOI: https://doi.org/10.21203/rs.3.rs-31889/v1

License: @ (i) This work is licensed under a Creative Commons Attribution 4.0 International License. Read Full License 


\section{Abstract}

\section{Background}

The relationship between left ventricular ejection fraction (LVEF) and cardiovascular (CV) outcome is well documented in patients with low LVEF. Ventilatory inefficiency is an important prognostic predictor in all spectrums of heart failure (HF). In stable HF outpatients, whether the relationship between LVEF and CV outcome is affected by ventilatory inefficiency remains unknown. We hypothesized that the presence of ventilatory inefficiency influences the prognostic predictability of LVEF in chronic HF outpatients.

Materials and Methods

In total, $169 \mathrm{HF}$ outpatients underwent the cardiopulmonary exercise test (CPET) and were followed up for a median of 9.25 years. Subjects were divided into five groups of similar size according to baseline LVEF ( $£ 39 \%$, $40-58 \%, 59-68 \%, 69-74 \%$, and ${ }^{3} 75 \%$ ). The primary endpoints were CV mortality and first HF hospitalization. The Cox proportional hazard model was used for simple and multiple regression analyses to evaluate the interrelationship between LVEF and ventilatory inefficiency (ventilatory equivalent for carbon dioxide [VE/VCO2] at anaerobic threshold [AT] $>34.3$, optimized cut-point).

Results

Only LVEF and VE/VCO2 at AT were significant predictors of major CV events. The lower LVEF subgroup (LVEF $£ 39 \%$ ) was associated with an increased risk of CV mortality or HF hospitalization relative to the LVEF $375 \%$ subgroup except for patients with ventilatory inefficiency $(p=0.400)$. The interaction effect between LVEF and ventilatory inefficiency was not significant $(p=0.579)$.

\section{Conclusions}

Ventilatory inefficiency influenced the prognostic predictability of LVEF in reduced LVEF outpatients. Ventilatory inefficiency can be used as the therapeutic target in HF management.

Trial registration

ClinicalTrials.gov (identifier: NCT04141345), 10/24/2019, retrospectively registered

\section{Introduction}

Heart failure (HF) is a leading cause of cardiovascular (CV) mortality and hospitalization. Preventing hospitalization in HF patients, such as using a multidisciplinary treatment strategy, has become a great priority for clinicians, researchers, and policymakers [1]. In addition to clinical demographic risk factors, left ventricular ejection fraction (LVEF) determined by echocardiography is the most commonly used parameter for the diagnosis and management of stable chronic HF patients [2, 3]. The relationship between LVEF and CV outcome is well documented in patients with low LVEF HF[4]. However, LVEF is less useful as a prognostic indicator when it is $>45 \%[5,6]$. Thus, reliable assessment of prognosis and risk stratification remain a challenge in HF outpatients across the full spectrum of LVEF. 
The cardiopulmonary exercise test (CPET) is a useful tool in all stages of HF patient management, from diagnosis to risk assessment [7]. In the past several decades, the peak oxygen uptake (peak VO2/kg) from CPET was considered as the best predictor of 1- to 3-year event-free survival after HF [8]. In some patients, ventilatory inefficiency during exercise may be a superior predictor of prognosis compared to peak V02/kg [9, 10].

Pulmonary abnormalities, such as impaired lung mechanics and abnormal alveolar-capillary gas exchange, may be caused by respiratory comorbidities or HF itself [11]. In stable HF outpatients, whether the relationship between LVEF and CV outcome is affected by ventilatory inefficiency remains unknown. In this study, we hypothesized that the presence of ventilatory inefficiency influences the prognostic predictability of LVEF in stable chronic HF patients.

\section{Methods}

\section{Subjects}

A cohort of $169 \mathrm{HF}$ outpatients with exercise intolerance took the CPET at a tertiary referral center between May 2007 and July 2010. Patients with concurrent signs and symptoms of HF (New York Heart Association functional class II IV) and evidence of structural heart disease (increased left atrial size or left ventricle hypertrophy) were recruited consecutively. Diagnosis was established by the attending physicians. Ischemic cardiomyopathy was defined as HF with the presence of severe coronary artery disease or a history of myocardial infarction. Valvular cardiomyopathy was defined as HF caused by primary disease of one of the four heart valves. Dilated cardiomyopathy was defined as dilation and impaired left ventricle contraction, in which primary and secondary causes of heart disease (e.g., coronary artery disease and myocarditis) were excluded. Patients who had a history of HF hospitalization within 6 months or are unable to perform an exercise test were excluded from the study. The patients were followed up at a median of 9.25 years (interquartile range [IQR], $7.48 \sim 10.32$ years) since the administration of CPET. LVEF was assessed by quantitative echocardiography. This study was approved by the Institutional Review Board of the Kaohsiung Chang Gung Memorial Hospital and was conducted in accordance with the Helsinki Declaration of 1975 (as revised in 1983). This study was registered at ClinicalTrials.gov (identifier: NCT04141345). Informed consent was obtained prior to CPET administration in all subjects.

\section{CPET procedures}

Patients performed an upright graded bicycle exercise using an individualized protocol. The heart rate was continuously monitored by electrocardiography at rest and during exercise. Blood pressure was measured using an electronic sphygmomanometer every 2 minutes and as needed. The minute ventilation (VE), oxygen consumption (VO2), and carbon dioxide production (VCO2) were continuously recorded every 1 minute using a respiratory mass spectrometer (Vmax Encore, VIASYS, Yorba Linda, CA, USA). Prior to each respiratory gas analysis study, the mass spectrometer was calibrated with a standard gas of known concentration. The peak $\mathrm{VO} 2 / \mathrm{kg}$ and the peak respiratory exchange ratio (RER) were defined as the highest 30 -second average value obtained during exercise. The anaerobic threshold (AT) was determined using the V-slope method. The 
VE/VCO2 at AT was calculated as the average VE/VCO2 for 1 minute during AT and immediately after AT. If the AT could not be determined, the lowest VE/VCO2 was determined by averaging the three lowest consecutive 0.5 -minute data points. Since the variability of VE/VCO2 at AT is slightly lower than the variability of the slope of VE versus VCO2 below the ventilatory compensatory point $[12,13]$, this study used VE/VCO2 at AT as a marker of ventilatory efficiency. Spirometric measurements included lung vital capacity, forced vital capacity, forced expiratory volume in 1 second, and maximal voluntary ventilation.

The criteria for discontinuing the test were as follows: request by the subject, threatened arrhythmia, peak RER $>1.1$, and $\geq 2.0 \mathrm{~mm}$ of horizontal or downslope ST segment depression during progressive exercise. The CPET exams were conducted by a qualified physical therapist under the supervision of a physician.

\section{Outcome analysis}

Defined time-dependent CV outcomes included CV mortality and first HF hospitalization, which were the primary endpoints of the analysis. Study subjects were followed up until the end of 2018. HF hospitalization was defined as an unplanned hospitalization due to new or worsening $\mathrm{HF}$ requiring the use of intravenous diuretics, inotropes, or vasodilators.

\section{Statistical analyses}

Subjects were divided into five groups of similar size according to baseline LVEF ( $\leq 39 \%, 40-58 \%, 59-68 \%$, $69-74 \%$, and $\geq 75 \%$ ) to evaluate the relationship between LVEF and CV outcomes. Comparisons between LVEF groups were analyzed using Pearson's chi-square test or Fisher's exact test for categorical variables. Continuous variables were expressed as median (IQR). Comparisons between LVEF groups were analyzed using the Kruskal-Wallis test and multiple comparisons for continuous variables. The Kolmogorov-Smirnov test was used to test for normality. For the univariate and multivariable analyses, the hazard ratio and $95 \%$ confidence interval were computed using the Cox proportional hazard model. The primary endpoint was defined as CV mortality or the first HF hospitalization. The various CPET parameters were evaluated as predictors of primary endpoints by performing time-dependent receiver operating characteristic curve (ROC) analyses. Optimized threshold values for VE/VCO2 at AT were identified via ROC analysis and the Youden index. The Cox proportional hazard model was used for simple and multiple regression analyses to evaluate the interrelationship between LVEF and ventilatory inefficiency (defined as VE/VCO2 at AT > 34.3, optimized cutoff point). The interaction term "ventilatory inefficiency multiplied by LVEF category" was introduced to the previous model. Data were analyzed using R v3.6.1 software using "time ROC" and "survival" package and SPSS 22.0 (SPSS Inc., Chicago, IL, USA). In all analyses, a p value less than 0.05 was considered statistically significant.

\section{Results}

The mean LVEF in our HF outpatients was $64.0 \pm 18.6 \%$. The baseline clinical demographic and pharmacological characteristics according to LVEF are shown in Table I. Patients with higher ejection fraction (EF) were more often female and more likely to have a history of hypertension. Patients with lower EF were 
more likely to have a smoking history, have received coronary intervention, and have ischemic cardiomyopathy. Patients who suffered from dilated cardiomyopathy had lower EF. The incidence of diabetes, valvular heart disease, and ischemic stroke did not differ across these LVEF subgroups. The distribution of age also did not differ significantly across the LVEF subgroups. The proportion of patients who received betablockers, angiotensin-converting enzyme inhibitors (ACEls), angiotensin-receptor blockers, loop diuretics, and mineralocorticoid receptor antagonists (MRAs) increased in the lower EF patients. In contrast, the proportion of patients who received dihydropyridine calcium channel blockers increased in the higher EF patients. The CPET parameters including peak VO2/kg, AT, and VE/VCO2 at AT had a significant difference across the spectrum of LVEF (Table 1). 
Table 1

Baseline clinical and pharmacological characteristics by LVEF

\begin{tabular}{|c|c|c|c|c|c|c|c|}
\hline variables & $\begin{array}{l}\text { All patients } \\
(\mathrm{n}=169)\end{array}$ & $\begin{array}{l}\text { LVEF } \\
\leq 39 \%(37)\end{array}$ & $\begin{array}{l}\text { LVEF } \\
40-58 \% \\
(31)\end{array}$ & $\begin{array}{l}\text { LVEF } \\
59- \\
68(38) \%\end{array}$ & $\begin{array}{l}\text { LVEF } \\
69-74 \% \\
(32)\end{array}$ & $\begin{array}{l}\text { LVEF } \\
\geq 75 \%(31)\end{array}$ & $\begin{array}{l}p \\
\text { value }\end{array}$ \\
\hline age & $55.7 \pm 13.5$ & $50.9 \pm 14.7$ & $59.6 \pm 12.3$ & $54.3 \pm 12.8$ & $57.1 \pm 14.6$ & $57.7 \pm 11.7$ & 0.097 \\
\hline male & $121(71.6 \%)$ & $34(91.9 \%)$ & $23(74.2 \%)$ & $27(71.1 \%)$ & $17(53.1 \%)$ & $20(64.5 \%)$ & 0.008 \\
\hline $\begin{array}{l}\text { Hypertension } \\
(\%)\end{array}$ & $99(58.6 \%)$ & $13(35.1 \%)$ & $23(74.2 \%)$ & $23(60.5 \%)$ & $19(65.5 \%)$ & $21(72.4 \%)$ & 0.006 \\
\hline Diabetes (\%) & $37(22.7 \%)$ & $9(24.3 \%)$ & $10(32.3 \%)$ & $10(26.3 \%)$ & $4(13.8 \%)$ & $4(14.3 \%)$ & 0.355 \\
\hline Smoking (\%) & $39(23.5 \%)$ & $16(43.2 \%)$ & $8(25.8 \%)$ & 7(18.4) & $5(16.1 \%)$ & $3(10.3 \%)$ & 0.015 \\
\hline $\begin{array}{l}\text { Ischemic } \\
\text { stroke (\%) }\end{array}$ & $9(5.6 \%)$ & $0(0 \%)$ & $1(3.2 \%)$ & $2(5.3 \%)$ & $2(6.9 \%)$ & $4(14.3 \%)$ & 0.158 \\
\hline $\begin{array}{l}\text { Ischemic CM } \\
(\%)\end{array}$ & $33(19.5 \%)$ & $15(40.5 \%)$ & $10(32.3 \%)$ & $2(5.3 \%)$ & $4(12.5 \%)$ & $2(6.5 \%)$ & $\begin{array}{l}< \\
0.0001\end{array}$ \\
\hline $\begin{array}{l}\text { Valvular CM } \\
(\%)\end{array}$ & $22(13.0 \%)$ & $3(8.1 \%)$ & $6(19.4 \%)$ & $4(10.5 \%)$ & $3(9.4 \%)$ & $6(19.4 \%)$ & 0.497 \\
\hline $\begin{array}{l}\text { Dilated CM } \\
(\%)\end{array}$ & $24(14.2 \%)$ & $15(40.5 \%)$ & $7(22.6 \%)$ & $2(5.3 \%)$ & $0(0 \%)$ & $0(0 \%)$ & $<0.0001$ \\
\hline Prior PCI (\%) & $29(17.2 \%)$ & $13(35.1 \%)$ & $9(29.0 \%)$ & $2(5.3 \%)$ & $4(12.5 \%)$ & $1(3.2 \%)$ & 0.001 \\
\hline \multicolumn{8}{|l|}{ Medication } \\
\hline $\begin{array}{l}\text { Beta-blocker } \\
(\%)\end{array}$ & $97(58.4 \%)$ & $30(81.1 \%)$ & $25(80.6 \%)$ & $19(50.0 \%)$ & $12(38.7 \%)$ & $11(37.9 \%)$ & $\begin{array}{l}< \\
0.0001\end{array}$ \\
\hline $\begin{array}{l}\text { ACEI/ARB } \\
(\%)\end{array}$ & $114(67.5 \%)$ & $32(86.5 \%)$ & $28(90.3 \%)$ & $21(55.3 \%)$ & $15(46.9 \%)$ & $18(58.1 \%)$ & $\stackrel{<}{0.0001}$ \\
\hline $\begin{array}{l}\text { DHP Ca } \\
\text { channel } \\
\text { blocker (\%) }\end{array}$ & $36(21.7 \%)$ & $1(2.7 \%)$ & $10(32.3 \%)$ & $5(13.2 \%)$ & $11(35.5 \%)$ & $9(31.0 \%)$ & 0.002 \\
\hline $\begin{array}{l}\text { Loop } \\
\text { diuretics (\%) }\end{array}$ & $43(25.9 \%)$ & $22(59.5 \%)$ & $13(41.9 \%)$ & $3(7.9 \%)$ & $4(12.9 \%)$ & $1(3.4 \%)$ & $<0.0001$ \\
\hline MRA (\%) & $21(12.4 \%)$ & $13(35.1 \%)$ & $5(16.1 \%)$ & $2(5.3 \%)$ & $1(3.2 \%)$ & $0(0 \%)$ & $<.0001$ \\
\hline Statin (\%) & $53(31.9 \%)$ & $13(35.1 \%)$ & $9(29.0 \%)$ & $12(31.6 \%)$ & $10(32.3 \%)$ & $9(31.0 \%)$ & 0.989 \\
\hline
\end{tabular}

LVEF: left ventricle ejection fraction, CM: cardiomyopathy, PCl: percutaneous coronary intervention, ACEl: angiotensin-converting enzyme inhibitor, ARB: angiotensin receptor blocker, DHP Ca channel blocker: Dihydropyridine calcium channel blocker, MRA: mineralocorticoid receptor antagonist, CPET: cardiopulmonary exercise test, VO2/kg: oxygen consumption per kilogram, VE: minute ventilation, AT: anaerobic threshold, $\mathrm{VE} / \mathrm{VCO}_{2}$ at $\mathrm{AT}$ : ventilatory equivalent for carbon dioxide at anaerobic threshold, RER: respiratory exchange ratio. 


\begin{tabular}{|c|c|c|c|c|c|c|c|}
\hline \multirow[t]{2}{*}{ variables } & \multirow{2}{*}{$\begin{array}{l}\text { All patients } \\
(n=169)\end{array}$} & \multirow{2}{*}{$\begin{array}{l}\text { LVEF } \\
\leq 39 \%(37)\end{array}$} & \multirow{2}{*}{$\begin{array}{l}\text { LVEF } \\
40-58 \% \\
(31)\end{array}$} & \multirow{2}{*}{$\begin{array}{l}\text { LVEF } \\
59- \\
68(38) \%\end{array}$} & \multirow{2}{*}{$\begin{array}{l}\text { LVEF } \\
69-74 \% \\
(32)\end{array}$} & \multirow{2}{*}{$\begin{array}{l}\text { LVEF } \\
\geq 75 \%(31)\end{array}$} & \multirow{2}{*}{$\begin{array}{l}p \\
\text { value }\end{array}$} \\
\hline & & & & & & & \\
\hline \multicolumn{8}{|l|}{$\begin{array}{l}\text { Parameters } \\
\text { of CPET }\end{array}$} \\
\hline $\begin{array}{l}\text { Peak } 02 \\
\text { pulse } \\
\text { (ml/beat) }\end{array}$ & $\begin{array}{l}11.9(9.64 \\
\sim 14.89)\end{array}$ & $\begin{array}{l}11.04 \\
(9.18 \sim \\
15.99)\end{array}$ & $\begin{array}{l}10.97 \\
(7.78 \sim \\
13.76)\end{array}$ & $\begin{array}{l}12.16 \\
(9.93 \sim \\
14.92)\end{array}$ & $\begin{array}{l}12.11 \\
(9.42 \sim \\
15.1)\end{array}$ & $\begin{array}{l}12.12 \\
(10.11 \sim \\
14.90)\end{array}$ & 0.303 \\
\hline $\begin{array}{l}\text { Peak VO2/kg } \\
(\mathrm{ml} / \mathrm{kg} / \mathrm{min})\end{array}$ & $\begin{array}{l}22.9(18.2 \\
\sim 28.4)\end{array}$ & $\begin{array}{l}20.0 \\
(15.9 \sim \\
26.0)\end{array}$ & $\begin{array}{l}21.3 \\
(16.8 \sim \\
25.1)\end{array}$ & $\begin{array}{l}25.1 \\
(19.1 \sim \\
29.7)\end{array}$ & $\begin{array}{l}23.4 \\
(19.5 \sim \\
29.0)\end{array}$ & $\begin{array}{l}25.5 \\
(19.4 \sim \\
31.9)\end{array}$ & 0.045 \\
\hline $\begin{array}{l}\text { Peak VE } \\
\text { (L/min) }\end{array}$ & $\begin{array}{l}54.0(43.0 \\
\sim 65.0)\end{array}$ & $\begin{array}{l}60.0 \\
(44.5 \sim \\
71.0)\end{array}$ & $\begin{array}{l}52.0 \\
(37.0 \sim \\
63.0)\end{array}$ & $\begin{array}{l}59.0 \\
(45.8 \sim \\
68.8)\end{array}$ & $\begin{array}{l}49.0 \\
(41.0 \sim \\
60.5)\end{array}$ & $\begin{array}{l}49.0 \\
(43.0 \sim \\
65.0)\end{array}$ & 0.159 \\
\hline $\begin{array}{l}\text { AT (\% of VO2 } \\
\text { max) }\end{array}$ & $\begin{array}{l}54.9(45.8 \\
\sim 66.2)\end{array}$ & $\begin{array}{l}50.0 \\
(41.2 \sim \\
60.7)\end{array}$ & $\begin{array}{l}51.0 \\
(45.7 \sim \\
57.8)\end{array}$ & $\begin{array}{l}58.2 \\
(49.2 \sim \\
66.4)\end{array}$ & $\begin{array}{l}56.4 \\
(44.6 \sim \\
73.5)\end{array}$ & $\begin{array}{l}61.7 \\
(52.2 \sim \\
74.2)\end{array}$ & 0.007 \\
\hline $\begin{array}{l}\text { VE/VCO2 at } \\
\text { AT }\end{array}$ & $\begin{array}{l}32.3(29.2 \\
\sim 35.8)\end{array}$ & $\begin{array}{l}33.4 \\
(29.9 \sim \\
38.1)\end{array}$ & $\begin{array}{l}34.8 \\
(29.8 \sim \\
37.9)\end{array}$ & $\begin{array}{l}31.7 \\
(28.8 \sim \\
35.8)\end{array}$ & $\begin{array}{l}32.0 \\
(28.9 \sim \\
34.1)\end{array}$ & $\begin{array}{l}30.9 \\
(27.7 \sim \\
33.1)\end{array}$ & 0.036 \\
\hline Peak RER & $\begin{array}{l}1.04(0.98 \\
\sim 1.09)\end{array}$ & $\begin{array}{l}1.05 \\
(1.02 \sim \\
1.12)\end{array}$ & $\begin{array}{l}1.02 \\
(0.97 \sim \\
1.09)\end{array}$ & $\begin{array}{l}1.05(1.0 \\
\sim 1.12)\end{array}$ & $\begin{array}{l}1.03 \\
(0.96 \sim \\
1.07)\end{array}$ & $\begin{array}{l}1.04 \\
(0.95 \sim \\
1.07)\end{array}$ & 0.118 \\
\hline $\begin{array}{l}\text { Peak VO2 } \\
\text { (L/min) }\end{array}$ & $\begin{array}{l}1600 \\
(1233 \sim \\
2074)\end{array}$ & $\begin{array}{l}1528 \\
(1101 \sim \\
2217)\end{array}$ & $\begin{array}{l}1461 \\
(980 \sim \\
1676)\end{array}$ & $\begin{array}{l}1668 \\
(1352 \sim \\
2114)\end{array}$ & $\begin{array}{l}1609 \\
(1245 \sim \\
1982)\end{array}$ & $\begin{array}{l}1706 \\
(1339 \sim \\
2117)\end{array}$ & 0.152 \\
\hline $\begin{array}{l}\text { Peak Work } \\
\text { (Watts) }\end{array}$ & $\begin{array}{l}119.0 \\
(77.5 \sim \\
161.5)\end{array}$ & $\begin{array}{l}135.0 \\
(69.0 \sim \\
193.5)\end{array}$ & $\begin{array}{l}96.0 \\
(74.0 \sim \\
125.0)\end{array}$ & $\begin{array}{l}125.5 \\
(88.5 \sim \\
162.3)\end{array}$ & $\begin{array}{l}115.5 \\
(79.8 \sim \\
158.5)\end{array}$ & $\begin{array}{l}123.0 \\
(69.0 \sim \\
158.0)\end{array}$ & 0.353 \\
\hline $\begin{array}{l}\text { Breathing } \\
\text { Reserve (L) }\end{array}$ & $\begin{array}{l}28.9(15.1 \\
\sim 42.0)\end{array}$ & $\begin{array}{l}34.0 \\
(12.8 \sim \\
44.2)\end{array}$ & $\begin{array}{l}26.2 \\
(10.6 \sim \\
40.0)\end{array}$ & $\begin{array}{l}30.9 \\
(22.0 \sim \\
42.9)\end{array}$ & $\begin{array}{l}20.2(8.5 \\
\sim 35.6)\end{array}$ & $\begin{array}{l}33.2 \\
(18.2 \sim \\
41.6)\end{array}$ & 0.221 \\
\hline \multicolumn{8}{|c|}{$\begin{array}{l}\text { LVEF: left ventricle ejection fraction, CM: cardiomyopathy, PCl: percutaneous coronary intervention, ACEI: } \\
\text { angiotensin-converting enzyme inhibitor, ARB: angiotensin receptor blocker, DHP Ca channel blocker: } \\
\text { Dihydropyridine calcium channel blocker, MRA: mineralocorticoid receptor antagonist, CPET: } \\
\text { cardiopulmonary exercise test, VO2/kg: oxygen consumption per kilogram, VE: minute ventilation, AT: } \\
\text { anaerobic threshold, VE/VCO }{ }_{2} \text { at AT: ventilatory equivalent for carbon dioxide at anaerobic threshold, RER: } \\
\text { respiratory exchange ratio. }\end{array}$} \\
\hline
\end{tabular}

Within a median follow-up period of 9.25 years (IQR, $7.48 \sim 10.32$ years), 49 patients had achieved our primary endpoints. The relationship between LVEF and the major cardiac events, including CV mortality, is shown in Table 2. The risk of major CV events and CV mortality was increased in the lower LVEF subgroups $(p=0.002$ and 0.001 , respectively). Table III shows that, according to the univariate Cox regression analysis, the significant predictors of major CV events included comorbid lung disease, diabetes, smoking, LVEF, dilated cardiomyopathy, and treatment with beta-blockers, loop diuretics, or MRAs. The CPET parameters including 
$\mathrm{VE} / \mathrm{VCO} 2$ at $\mathrm{AT}, \triangle \mathrm{VO} 2 / \triangle \mathrm{WR}$, peak 02 pulse, peak V02, peak VO2/kg, peak work, and AT were significant predictors for major $\mathrm{CV}$ events based on the univariate analysis. In the multivariate Cox regression analyses, only LVEF and VE/VCO2 at AT were found to be significant predictors of major CV events in our cohort study (Table 3). The optimized threshold value of VE/VCO2 at AT was identified by ROC analysis. For predicting major CV events in all patients, the best cutoff point for VE/VCO2 at AT was 34.3 (64.3 sensitivity and 22.0\% specificity, Youden index $=0.42$ ).

Table 2

outcomes by LVEF

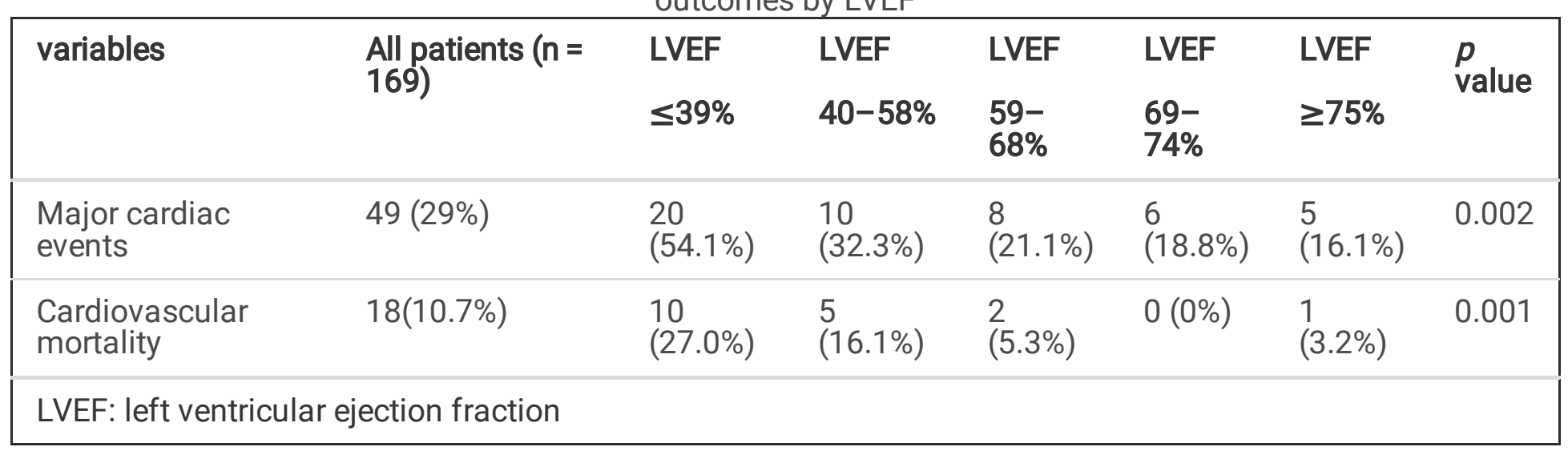


Table 3

Univariate and multivariate analysis of predictors of major cardiovascular events

\begin{tabular}{|c|c|c|c|c|c|c|}
\hline \multirow[t]{2}{*}{ Independent variable } & \multicolumn{3}{|c|}{ Univariate analysis } & \multicolumn{3}{|c|}{ Multivariate analysis } \\
\hline & HR & $(95 \% \mathrm{Cl})$ & $p$-value & HR & $(95 \% \mathrm{Cl})$ & $p$-value \\
\hline Age at CPET & 1.0 & $(0.99-1.02)$ & .966 & & & \\
\hline Male & 1.66 & $(0.83-3.33)$ & .152 & & & \\
\hline \multicolumn{7}{|l|}{ Lung Disease } \\
\hline Obstructive & 1.45 & $(0.57-3.65)$ & .433 & & & \\
\hline Restrictive & 1.73 & $(0.99-3.02)$ & .057 & & & \\
\hline Both & 1.92 & $(1.09-3.40)$ & .025 & & & \\
\hline Ischemic stroke & 1.56 & $(0.56-4.44)$ & .392 & & & \\
\hline Myocardial infarction & 1.31 & $(0.66-2.63)$ & .442 & & & \\
\hline Hypertension & 0.66 & $(0.37-1.15)$ & .139 & & & \\
\hline Revascularization & 1.74 & $(0.91-3.34)$ & .096 & & & \\
\hline Diabetes & 2.06 & $(1.14-3.71)$ & .016 & & & \\
\hline Smoking & 1.97 & $(1.10-3.56)$ & .024 & & & \\
\hline LVEF & 0.97 & $(0.96-0.98)$ & $<.001$ & 0.98 & $(0.96-0.99)$ & .002 \\
\hline Ischemic cardiomyopathy & 1.65 & $(0.88-3.11)$ & .122 & & & \\
\hline Dilated cardiomyopathy & 2.03 & $(1.04-3.98)$ & .039 & & & \\
\hline Valvular cardiomyopathy & 1.37 & $(0.64-2.92)$ & .416 & & & \\
\hline Beta-blocker & 2.24 & $(1.19-4.22)$ & .013 & & & \\
\hline ACEI/ARB & 1.88 & $(0.96-3.69)$ & .064 & & & \\
\hline$D H P \mathrm{Ca}^{+}$channel blocker & 0.88 & $(0.44-1.76)$ & .718 & & & \\
\hline Loop diuretic & 3.39 & $(1.93-5.96)$ & $<.001$ & & & \\
\hline Spironolactone & 4.10 & $(2.17-7.77)$ & $<.001$ & & & \\
\hline Statin & 1.57 & $(0.89-2.78)$ & .121 & & & \\
\hline VE/VCO2 at AT & 1.19 & $(1.14-1.25)$ & $<.001$ & 1.17 & $(1.12-1.23)$ & $<.001$ \\
\hline$\Delta \mathrm{VO} 2 / \Delta \mathrm{WR}(\mathrm{ml} / \mathrm{min} / \mathrm{W})$ & 1.04 & $(1.01-1.07)$ & .008 & & & \\
\hline
\end{tabular}

HR: Hazard ratio, Cl: confidence interval, CPET: cardiac pulmonary exercise test, ACEl: angiotensinconverting enzyme inhibitor, ARB: angiotensin receptor blocker, DHP: dihydropyridine, $\mathrm{VE} / \mathrm{VCO}_{2}$ at AT: ventilatory equivalent for carbon dioxide at anaerobic threshold, $\triangle \mathrm{VO} 2 / \triangle \mathrm{WR}$ : the ratio of increase in oxygen uptake to increase in work rate, peak VO2: peak oxygen consumption, RER: respiratory exchange ratio, VE: minute ventilation, V02/kg: oxygen consumption per kilogram, AT: anaerobic threshold. 


\begin{tabular}{|c|c|c|c|c|c|c|}
\hline \multirow[t]{2}{*}{ Independent variable } & \multicolumn{3}{|c|}{ Univariate analysis } & \multicolumn{3}{|c|}{ Multivariate analysis } \\
\hline & $H R$ & $(95 \% \mathrm{Cl})$ & $p$-value & HR & $(95 \% \mathrm{Cl})$ & $p$-value \\
\hline Peak 02 pulse (ml/beat) & 0.90 & $(0.83-0.97)$ & 0.009 & & & \\
\hline Peak V02 (L/min) & 1.0 & $(0.99-1.0)$ & .001 & & & \\
\hline Peak RER & 0.27 & $(0.01-5.60)$ & .395 & & & \\
\hline Breathing reserve $(\mathrm{ml})$ & 1.00 & $(0.99-1.01)$ & .934 & & & \\
\hline Peak VE (L/mins) & 1.0 & $(0.98-1.01)$ & .731 & & & \\
\hline Peak V02/kg (ml/kg/mins) & 0.90 & $(0.85-0.95)$ & $<.001$ & & & \\
\hline Peak work (Watt) & 0.99 & $(0.99-1.0)$ & .009 & & & \\
\hline Anaerobic threshold & 0.95 & $(0.93-0.97)$ & $<.001$ & & & \\
\hline \multicolumn{7}{|c|}{$\begin{array}{l}\text { HR: Hazard ratio, Cl: confidence interval, CPET: cardiac pulmonary exercise test, ACEI: angiotensin- } \\
\text { converting enzyme inhibitor, ARB: angiotensin receptor blocker, DHP: dihydropyridine, VE/VCO }{ }_{2} \text { at AT: } \\
\text { ventilatory equivalent for carbon dioxide at anaerobic threshold, } \triangle \mathrm{VO} 2 / \triangle \mathrm{WR} \text { : the ratio of increase in } \\
\text { oxygen uptake to increase in work rate, peak VO2: peak oxygen consumption, RER: respiratory exchange } \\
\text { ratio, VE: minute ventilation, VO2/kg: oxygen consumption per kilogram, AT: anaerobic threshold. }\end{array}$} \\
\hline
\end{tabular}

As presented in Fig. 1, the relationship between LVEF and major CV events was not linear. We defined ventilatory inefficiency as VE/VCO2 at AT > 34.3. To characterize the relationship between LVEF and the risk of $\mathrm{CV}$ mortality or HF hospitalization among patients with ventilatory inefficiency, subjects were divided into five subgroups according to baseline LVEF. Figure 2 shows the relationship between LVEF and major CV events in patients with ventilatory inefficiency (VE/VCO2 at AT > 34.3) and in patients without ventilatory inefficiency (VE/VCO2 at AT $\leq 34.3$ ). After multivariable adjustment, the Cox proportional hazard model showed that the lower LVEF subgroup (LVEF $\leq 39 \%$ ) was associated with a significantly increased risk of CV mortality or HF hospitalization relative to the LVEF $\geq 75 \%$ subgroup among patients without ventilatory inefficiency (VE/VCO2 at AT $\leq 34.3)(p=0.019)$ and among all patients $(p=0.002)$ (Table 4$)$. Conversely, there was no prognostic predictability relative to low EF (LVEF $\leq 39 \%)$ among patients with ventilatory inefficiency (VE/VCO2 at AT > 34.3) $(p=0.400)$. However, the interaction effect between LVEF and ventilatory inefficiency in predicting CV major events was not significant $(p=0.579)$. 
Table 4

Adjust hazard ratio associated with LVEF for major cardiovascular events by baseline LVEF category relative to LVEF $\geq 75$

\begin{tabular}{|c|c|c|c|c|c|c|}
\hline & $\begin{array}{l}\mathrm{VE} / \mathrm{VCO} 2 \text { at } \mathrm{AT} \leq \\
34.3\end{array}$ & & $\begin{array}{l}\text { VE/VCO2 at AT > } \\
34.3\end{array}$ & & All & \\
\hline $\begin{array}{l}\text { LVEF } \\
\text { group }\end{array}$ & $\mathrm{HR}(95 \% \mathrm{Cl})$ & $\mathrm{p}$ & $\mathrm{HR}(95 \% \mathrm{Cl})$ & $\mathrm{p}$ & $\mathrm{HR}(95 \% \mathrm{Cl})$ & $\mathrm{p}$ \\
\hline$\leq 39$ & $12.00(1.50-96.01)$ & .019 & $1.63(0.52-5.08)$ & .400 & $\begin{array}{l}4.63(1.74- \\
12.35)\end{array}$ & .002 \\
\hline $40-58$ & $3.49(0.32-38.48)$ & .308 & $0.70(0.21-2.33)$ & .561 & $2.12(0.73-6.22)$ & .169 \\
\hline $59-68$ & $2.78(0.29-26.74)$ & .376 & $0.63(0.17-2.35)$ & .492 & $1.30(0.42-3.97)$ & .647 \\
\hline $69-74$ & $2.92(0.30-28.11)$ & .353 & $0.56(0.12-2.50)$ & .445 & $1.12(0.34-3.66)$ & .854 \\
\hline$\geq 75$ & 1 & & 1 & & 1 & \\
\hline
\end{tabular}

interaction term: $\mathrm{p}$ value $=0.579$

LVEF: left ventricular ejection fraction, HR: hazard ratio, VE/VCO2 at AT: ventilatory equivalent for carbon dioxide at anaerobic threshold

\section{Discussion}

In chronic HF outpatients followed for a median of 9.25 years, LVEF and VE/VCO2 at AT were both found to be significant independent predictors of increased risk of CV mortality or HF hospitalization. LVEF was a poor predictor in patients with ventilatory inefficiency and in those with LVEF > 40\%. Although our study showed that the interaction effect between LVEF and VE/VCO2 at AT was not significant, the prognostic predictability of LVEF was decreased in the HF with reduced LVEF (HFrEF, LVEF $\leq 39 \%$ ) population in the ventilatory inefficiency group. As demonstrated in the CHARM Program [5], the relationship between LVEF and CV outcomes is not linear. We also demonstrated a similar finding in chronic HF outpatients. This relationship was further diminished in the ventilatory inefficiency group. This phenomenon revealed that HF with preserve LVEF (HFpEF, LVEF $\geq 50 \%$ ) patients who had ventilatory inefficiency had similar CV outcomes as that of their HFrEF counterpart.

This study showed that the ventilation efficiency variable, in addition to LVEF, was a significant prognostic predictor in HF outpatients. Ventilatory inefficiency reflects the adverse effects of HF on lung mechanics and diffusion capacity [14]. An HF also augments ventilatory drive and increases hemodynamic demand associated with breathing work [15]. Ergoreceptors stimulate ventilation and activate sympathetic hormones in response to work. The ergoreflex in the muscle also affects ventilatory effort. In response to carbon dioxide and pulmonary $\mathrm{J}$ receptors (which likely respond to congestion and alveolar stiffness), central and pulmonary chemoreceptors contribute to the ergoreflex and result in excess ventilation [16]. In HF patients, a high ventilatory drive can reduce the partial pressure of $\mathrm{CO}_{2}(\mathrm{PaCO} 2)$ [17]. Consequently, a reduced $\mathrm{PaCO} 2$ and increased fractional dead space cause abnormally high VE/VCO2 at AT, i.e., ventilatory inefficiency [18, 19]. 
The mechanism of ventilatory inefficiency influences the outcomes of HF patients differently between the HFrEF and HFpEF patients. A study analyzed the ventilatory inefficiency between $24 \mathrm{HFrEF}$ patients and 33 HFpEF patients [20]. It demonstrated the loss of cardiac output augmentation related to ventilatory inefficiency regardless of LVEF; however, lung congestion parameters (echocardiographic parameter: e' and E/e') correlated with ventilatory inefficiency only in HFpEF. In another study, ventilatory inefficiency appears to be influenced by mechanisms regulating PaCO2 in HFrEF. In contrast, dead space to tidal volume ratio (VD/VT) plays a more important role in developing ventilatory inefficiency in HFpEF [21]. HFpEF and HFrEF may be two distinct entities in terms of ventilatory response to exercise; this study provides evidence that ventilatory inefficiency plays a critical role in HFpEF.

CPET-based measurements of ventilatory inefficiency provide unique physiologic information clinically relevant to contemporary treatment for HF. Several therapeutic interventions for HF affect ventilatory abnormalities both at rest and during exercise. For example, ACEl improves pulmonary diffusion, removes interstitial fluid, and improves pulmonary hemodynamic status [22]. Carvedilol, but not bisoprolol, improves ventilatory efficiency during exercise (reduction of VE/VCO2 slope and increase in maximum end-tidal $\mathrm{CO}_{2}$ pressure) [23]. Carvedilol may have direct effects on respiratory chemoreceptor activity based on the CARNEBI trial [24]. As ventilatory inefficiency is a significant prognostic predictor across the spectrum of LVEF, we should consider ventilatory abnormalities during exercise as therapeutic targets and treat them accordingly. Therapeutic interventions such as rehabilitation training (isolated quadriceps training) [25], device-guided paced breathing [26], yoga mantras [27], and reduction of afferent stimuli from ergopulmonary and cardiopulmonary receptors $[28,29]$ might all alleviate ventilatory inefficiency. The use of CPET-derived variables to guide therapy and improve outcome deserves further investigation.

This study has some limitations. First, the sample size was relatively small compared to those in other epidemiological studies. However, our study had a longer follow-up period than those of previous works. Second, patients were only recruited from outpatient clinics, which may have caused selection bias. The findings of this study may need further validation in other populations of patients with HF. Third, this study did not analyze other CPET variables that have been used to predict HF outcomes, e.g., oscillatory ventilation, endtidal $\mathrm{CO}_{2}$ pressure, $\mathrm{VO} 2$ kinetics during exercise, oxygen uptake efficiency slope, and heart rate recovery. Therefore, whether the predictive accuracy of these variables can be increased by combining them with VE/VCO2 at AT requires further investigation.

\section{Conclusions}

Ventilatory inefficiency influenced the prognostic predictability of LVEF in HFrEF patients when compared to patients with LVEF $\geq 75 \%$. The CPET-derived variable (VE/VCO2 at AT) can be used as the therapeutic target in $\mathrm{HF}$ management. However, the interaction effect between LVEF and ventilatory inefficiency in predicting CV outcomes was not significant.

\section{Declarations}

\section{Ethics approval and consent to participate}


This study was approved by the Institutional Review Board of the Kaohsiung Chang Gung Memorial Hospital (IRB No: 20171459B0) and was conducted in accordance with the Helsinki Declaration of 1975 (as revised in 1983). All the data obtained was anonymized. The need for consent was waived by the IRB of our hospital.

\section{Consent to publish}

Not applicable.

\section{Availability of data and materials}

All data generated or analyzed during this study are included in this published article. The datasets are available from the corresponding author on reasonable request.

\section{Competing interests}

The authors declare that the research was conducted in the absence of any commercial or financial relationships that could be construed as a potential conflict of interests.

\section{Funding}

We have no support or funding to report.

\section{Acknowledgments}

We thank Hsin-Yi Chien, Chih-Yun Lin, and the Biostatistics Center, Kaohsiung Chang Gung Memorial Hospital for statistics work.

\section{Authors' Contributions}

SMC led the conception and design of study, and revised the draft of the manuscript. LYW collected the research data and prepared the draft of the manuscript. PJW, MYL, and ANC performed clinical works and organized the collected data. YLC and THT performed the statistical analysis and drafted the manuscript. CLH and MCL supervised and validated the clinical works and results. All authors read and approved the final manuscript.

\section{References}

1. Chen SM, Fang YN, Wang LY, Wu MK, Wu PJ, Yang TH, Chen YL, Hang CL. Impact of multi-disciplinary treatment strategy on systolic heart failure outcome. BMC Cardiovasc Disord. 2019;19(1):220. 
2. Ponikowski P, Voors AA, Anker SD, Bueno H, Cleland JG, Coats AJ, Falk V, González-Juanatey JR, Harjola VP, Jankowska EA, et al. 2016 ESC Guidelines for the diagnosis and treatment of acute and chronic heart failure: The Task Force for the diagnosis and treatment of acute and chronic heart failure of the European Society of Cardiology (ESC). Developed with the special contribution of the Heart Failure Association (HFA) of the ESC. Eur J Heart Fail. 2016;18(8):891-975.

3. Yancy CW, Jessup M, Bozkurt B, Butler J, Casey DE, Drazner MH, Fonarow GC, Geraci SA, Horwich T, Januzzi JL, et al. 2013 ACCF/AHA guideline for the management of heart failure: executive summary: a report of the American College of Cardiology Foundation/American Heart Association Task Force on practice guidelines. Circulation. 2013;128(16):1810-52.

4. McDermott MM, Feinglass J, Lee PI, Mehta S, Schmitt B, Lefevre F, Gheorghiade M. Systolic function, readmission rates, and survival among consecutively hospitalized patients with congestive heart failure. Am Heart J. 1997;134(4):728-36.

5. Solomon SD, Anavekar N, Skali H, McMurray JJ, Swedberg K, Yusuf S, Granger CB, Michelson EL, Wang D, Pocock $S$, et al. Influence of ejection fraction on cardiovascular outcomes in a broad spectrum of heart failure patients. Circulation. 2005;112(24):3738-44.

6. Pocock SJ, Wang D, Pfeffer MA, Yusuf S, McMurray JJ, Swedberg KB, Ostergren J, Michelson EL, Pieper KS, Granger CB. Predictors of mortality and morbidity in patients with chronic heart failure. Eur Heart J. 2006;27(1):65-75.

7. Paolillo S, Agostoni P. Prognostic Role of Cardiopulmonary Exercise Testing in Clinical Practice. Ann Am Thorac Soc. 2017;14(Supplement_1):53-8.

8. Mehra MR, Canter CE, Hannan MM, Semigran MJ, Uber PA, Baran DA, Danziger-Isakov L, Kirklin JK, Kirk R, Kushwaha SS, et al. The 2016 International Society for Heart Lung Transplantation listing criteria for heart transplantation: A 10-year update. J Heart Lung Transplant. 2016;35(1):1-23.

9. Poggio R, Arazi HC, Giorgi M, Miriuka SG. Prediction of severe cardiovascular events by VE/VCO2 slope versus peak VO2 in systolic heart failure: a meta-analysis of the published literature. Am Heart J. 2010;160(6):1004-14.

10. Kleber FX, Vietzke G, Wernecke KD, Bauer U, Opitz C, Wensel R, Sperfeld A, Glaser S. Impairment of ventilatory efficiency in heart failure: prognostic impact. Circulation. 2000;101(24):2803-9.

11. Wasserman K, Zhang YY, Gitt A, Belardinelli R, Koike A, Lubarsky L, Agostoni PG. Lung function and exercise gas exchange in chronic heart failure. Circulation. 1997;96(7):2221-7.

12. Sun XG, Hansen JE, Garatachea N, Storer TW, Wasserman K. Ventilatory efficiency during exercise in healthy subjects. Am J Respir Crit Care Med. 2002;166(11):1443-8.

13. Guazzi M, Adams V, Conraads V, Halle M, Mezzani A, Vanhees L, Arena R, Fletcher GF, Forman DE, Kitzman DW, et al. EACPR/AHA Scientific Statement. Clinical recommendations for cardiopulmonary exercise testing data assessment in specific patient populations. Circulation. 2012;126(18):2261-74.

14. Myers J, Arena R, Cahalin LP, Labate V, Guazzi M. Cardiopulmonary Exercise Testing in Heart Failure. Curr Probl Cardiol. 2015;40(8):322-72.

15. Olson TP, Snyder EM, Johnson BD. Exercise-disordered breathing in chronic heart failure. Exerc Sport Sci Rev. 2006;34(4):194-201. 
16. Goodlin SJ. Palliative care in congestive heart failure. J Am Coll Cardiol. 2009;54(5):386-96.

17. Rocha A, Arbex FF, Sperandio PA, Souza A, Biazzim L, Mancuso F, Berton DC, Hochhegger B, Alencar MCN, Nery LE, et al. Excess Ventilation in Chronic Obstructive Pulmonary Disease-Heart Failure Overlap. Implications for Dyspnea and Exercise Intolerance. Am J Respir Crit Care Med. 2017;196(10):1264-74.

18. Johnson RL Jr. Gas exchange efficiency in congestive heart failure II. Circulation. 2001;103(7):916-8.

19. Johnson RL Jr. Gas exchange efficiency in congestive heart failure. Circulation. 2000;101(24):2774-6.

20. 10.1253/circrep.CR-20-0021

Tsujinaga S, Iwano H, Chiba Y, Ishizaka S, Sarashina M, Murayama M, Nakabachi M, Nishino H, Yokoyama S, Okada K, et al: Heart Failure With Preserved Ejection Fraction vs. Reduced Ejection Fractionã岖 - Mechanisms of Ventilatory Inefficiency During Exercise in Heart Failure - circulation reports 2020. https://doi.org/10.1253/circrep.CR-20-0021.

21. Van Iterson EH, Johnson BD, Borlaug BA, Olson TP. Physiological dead space and arterial carbon dioxide contributions to exercise ventilatory inefficiency in patients with reduced or preserved ejection fraction heart failure. Eur J Heart Fail. 2017;19(12):1675-85.

22. Guazzi M, Marenzi G, Alimento M, Contini M, Agostoni P. Improvement of alveolar-capillary membrane diffusing capacity with enalapril in chronic heart failure and counteracting effect of aspirin. Circulation. 1997;95(7):1930-6.

23. Agostoni P, Apostolo A, Cattadori G, Salvioni E, Berna G, Antonioli L, Vignati C, Schina M, Sciomer S, Bussotti $\mathrm{M}$, et al. Effects of beta-blockers on ventilation efficiency in heart failure. Am Heart J. 2010;159(6):1067-73.

24. Contini M, Apostolo A, Cattadori G, Paolillo S, lorio A, Bertella E, Salvioni E, Alimento M, Farina S, Palermo $\mathrm{P}$, et al. Multiparametric comparison of CARvedilol, vs. NEbivolol, vs. Blsoprolol in moderate heart failure: the CARNEBI trial. Int J Cardiol. 2013;168(3):2134-40.

25. Esposito F, Reese V, Shabetai R, Wagner PD, Richardson RS. Isolated quadriceps training increases maximal exercise capacity in chronic heart failure: the role of skeletal muscle convective and diffusive oxygen transport. J Am Coll Cardiol. 2011;58(13):1353-62.

26. Parati G, Malfatto G, Boarin S, Branzi G, Caldara G, Giglio A, Bilo G, Ongaro G, Alter A, Gavish B, et al. Device-guided paced breathing in the home setting: effects on exercise capacity, pulmonary and ventricular function in patients with chronic heart failure: a pilot study. Circ Heart Fail. 2008;1(3):178-83.

27. Bernardi L, Sleight P, Bandinelli G, Cencetti S, Fattorini L, Wdowczyc-Szulc J, Lagi A. Effect of rosary prayer and yoga mantras on autonomic cardiovascular rhythms: comparative study. BMJ. 2001;323(7327):1446-9.

28. Chua TP, Ponikowski PP, Harrington D, Chambers J, Coats AJ. Contribution of peripheral chemoreceptors to ventilation and the effects of their suppression on exercise tolerance in chronic heart failure. Heart. 1996;76(6):483-9.

29. Wensel R, Georgiadou P, Francis DP, Bayne S, Scott AC, Genth-Zotz S, Anker SD, Coats AJ, Piepoli MF. Differential contribution of dead space ventilation and low arterial pCO2 to exercise hyperpnea in patients with chronic heart failure secondary to ischemic or idiopathic dilated cardiomyopathy. Am J Cardiol. 2004;93(3):318-23. 


\section{Figures}

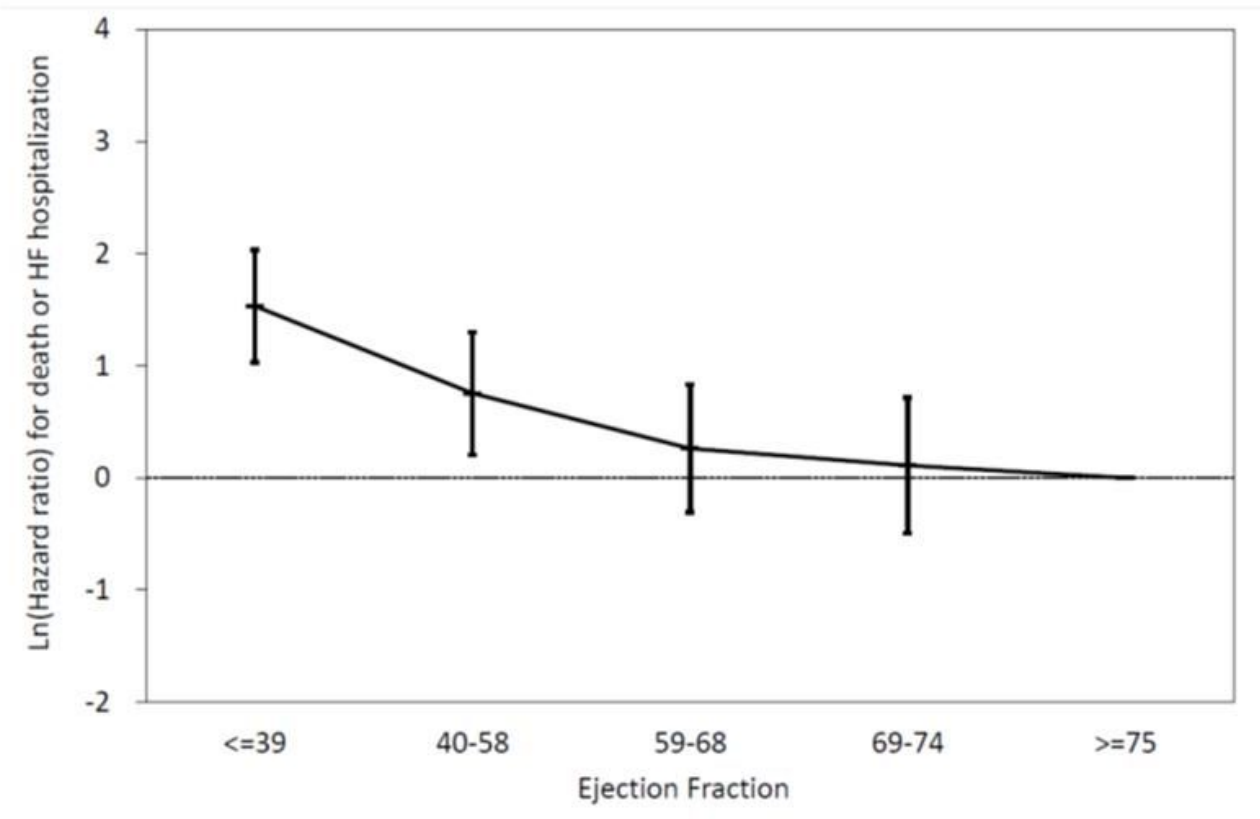

\section{Figure 1}

The relationship between LVEF and CV outcomes in all patients. This relationship was not linear. The lower LVEF subgroup (LVEF $\triangle 39 \%$ ) was associated with a significantly increased risk of CV mortality or HF hospitalization relative to the LVEF $\triangle 75 \%$ subgroup. $(p=0.002)$ Abbreviation: LVEF: left ventricular ejection fraction, CV: cardiovascular, HF: heart failure 


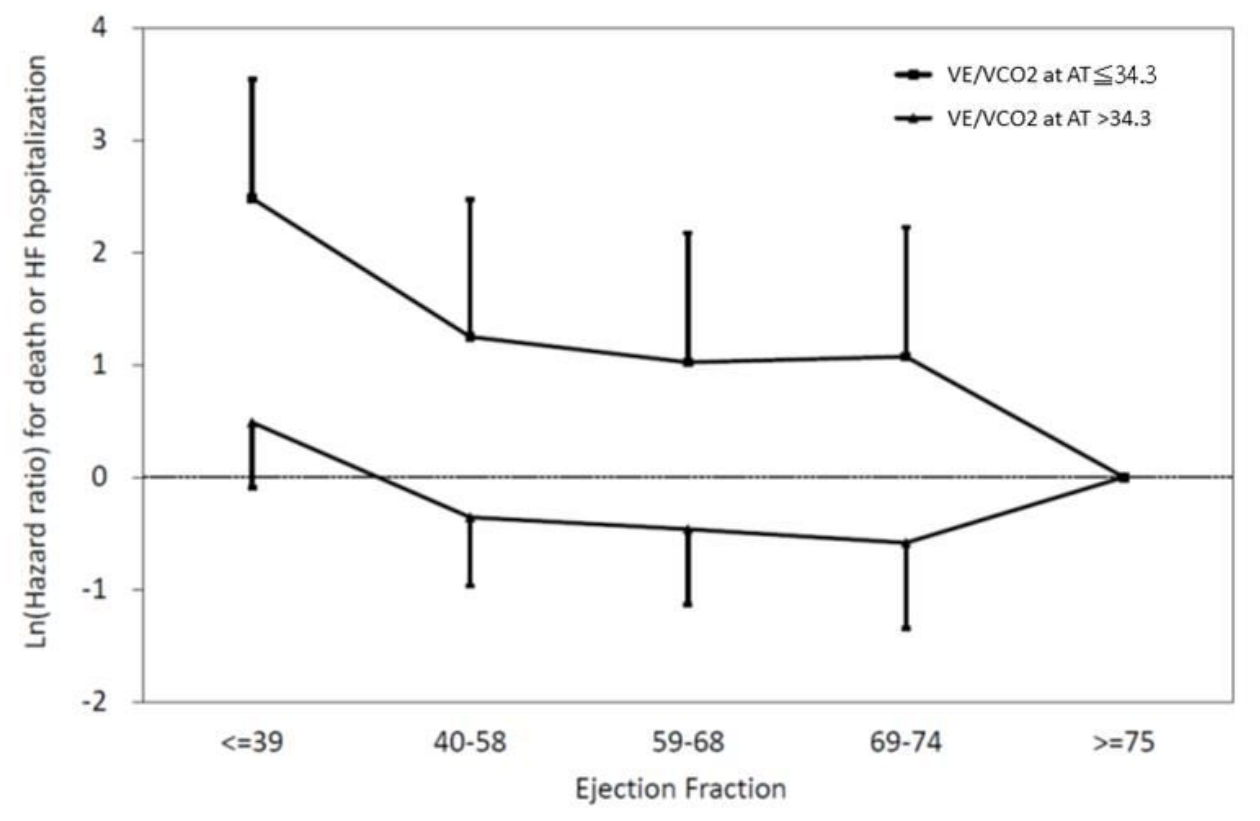

\section{Figure 2}

The relationship between LVEF and CV outcomes in patients with ventilatory inefficiency (VE/VCO2 at AT $>34.3$ ) and in patients without ventilatory inefficiency (VE/VCO2 at AT 『34.3). The lower LVEF subgroup (LVEF $\nabla 39 \%$ ) was associated with a significantly increased risk of CV mortality or HF hospitalization relative to the LVEF $\triangle 75 \%$ subgroup among patients without ventilatory inefficiency $(p=0.019)$. There was no prognostic predictability relative to low EF (LVEF $₫ 39 \%)$ among patients with ventilatory inefficiency $(p=0.400)$.

Abbreviation: LVEF: left ventricular ejection fraction, CV: cardiovascular, VE/VCO2 at AT: ventilatory equivalent for carbon dioxide at anaerobic threshold, HF: heart failure 